Original Research Article

\title{
A study of adverse cutaneous drug reactions in the department of dermatology of a teaching hospital in Jamnagar, India
}

\author{
Jagruti G. Dhanani, Anupama Sukhlecha*
}

Department of Pharmacology, M. P. Shah Govt. Medical College, Jamnagar, Gujarat, India

Received: 07 July 2017 Accepted: 29 July 2017

\section{*Correspondence to:}

Dr. Anupama Sukhlecha, Email: anupama_acad@ yahoo.co.in

Copyright: (C) the author(s), publisher and licensee Medip Academy. This is an openaccess article distributed under the terms of the Creative Commons Attribution NonCommercial License, which permits unrestricted noncommercial use, distribution, and reproduction in any medium, provided the original work is properly cited.

\begin{abstract}
Background: Adverse drug reactions cause significant morbidity and mortality in health care set up. They are responsible for a significant number of hospital admissions. The data for adverse cutaneous drug reactions (ACDRs) is limited our country. Thus, the present study emphasises on the need of an effective adverse drug reaction reporting programme.

Methods: A prospective study to assess the ACDRs in Dermatology department was conducted in a teaching hospital of India. Male to female ratio, most common class of drug, individual drugs causing ACDRs, common types of ACDRs were studied. Other parameters like causality assessment, preventability and seriousness of ACDRs were also studied.

Results: A majority of the patients (38\%) with ACDR were in the age group of 25-44 years followed by $45-64$ years $(31 \%)$. The rate of ACDRs was more in males (54\%). The most frequent ACDR reported was maculopapular rash (38\%), followed by urticaria (19\%) and fixed drug eruptions (12\%). Most of the reactions (93\%) were bizarre/ unpredictable in nature. Amongst drug groups, antimicrobials $(48 \%)$ were most frequently associated with ACDRs followed by NSAIDs (30\%) and antiepileptics (12\%). Most of the reactions (94\%) were mildmoderate in nature. Only $9 \%$ of ACDRs reported in this study were preventable. Paracetamol was the most common offending drug followed by cotrimoxazole, ibuprofen, amoxicillin, phenytoin in decreasing order. Causality assessment found ACDRs in probable category (68\%) using WHO-UMC criteria.

Conclusions: It is important to monitor and report adverse drug reactions in order to promote safe and rational use of medicines.
\end{abstract}

Keywords: Adverse cutaneous drug reactions, Causalty assessment, Fixed drug eruption, Maculopapular rash, Pharmacovigilance, Urticaria

\section{INTRODUCTION}

According to WHO an adverse drug reaction (ADR) is defined as a response to a drug which is noxious and unintended, which occurs at doses normally used in man for prophylaxis, diagnosis or therapy of disease or for modification of physiological function excluding failure to accomplish the intended purpose. ${ }^{1}$

ADRs cause of significant morbidity and mortality in patients. It has been estimated that one third to as high as one half of ADRs are preventable. They are responsible for a significant number of hospital admissions, among these; cutaneous ADRs (2-3\%) are one of the frequent reasons for patients to visit the clinicians. ${ }^{2}$ The incidence and severity of ADRs can be influenced by patient-related factors like age, sex, concurrent ailments, genetic factors, and drug related factors like type of drug, route of administration, duration of treatment, and dosage. The other important risk factors associated with adverse drug reactions are gender, increased number of medicine exposures, geriatric age, length of hospital stay and function of excreting organs. ${ }^{3}$ An adverse cutaneous drug reaction (ACDR) is any undesirable change in the structure or function of the skin, its appendages or mucous membranes and it encompasses all adverse events related to drug eruption, regardless of the etiology. ${ }^{4}$ Any skin 
disorder can be imitated, induced, or aggravated by drugs. Many of the commonly used medicines have reaction rates over $1 \% .^{5,6}$ There is a wide spectrum of cutaneous adverse drug reactions varying from transient maculopapular rash to fatal toxic epidermal necrolysis (TEN). ${ }^{7}$ The incidence of cutaneous drug eruptions is about $2.2 \%$ and is higher amongst inpatients and females. ${ }^{8}$ However, according to a drug surveillance programme, the incidence of cutaneous drug reactions varies from $15-30 \% .^{2}$ The incidence of ACDR in developed countries ranges from 1-3\% among in- patients whereas in developing countries like India some studies mention it to $2-5 \%$ of the inpatients but there is lack of comprehensive data amongst out-patients. ${ }^{2,9}$ There is no gold standard investigation for confirmation of a drug-induced reaction. Instead diagnosis and assessment of a drug cause involve analysis of a number of features such as timing of drug exposure and reaction time, course of reaction with drug withdrawal / discontinuation, timing and nature of a recurrent eruption on rechallenge, a history of similar reaction to the suspected drug and previous reports of similar reactions to the same drug. ${ }^{10}$

The objective of our study was to ascertain the clinical spectrum of ACDRs and the causative drugs, and to find any drug related risk factors in a teaching hospital in Jamnagar. It also emphasised on the need of an effective pharmacovigilance programme. ${ }^{11}$

\section{METHODS}

The study was carried out from April 2015 to March 2016 in the Department of Dermatology of Guru Gobind Singh Government Hospital, Jamnagar, a 1275 bedded teaching hospital attached to Shri M. P. Shah Govt. Medical College, Gujarat. Approval of the Institutional Ethics Committee was obtained before commencement of the study. All patients irrespective of age and sex suspected of having drug reactions seen during the period of one year were included in the study after taking their written consent.

In every case, a detailed history was elicited and a thorough clinical examination was carried out. To establish the etiologic agent for a particular type of reaction, attention was paid to the drug history, temporal correlation with the drug, duration of the rash, approximate incubation period, morphology of the eruption, associated mucosal or systemic involvement and improvement of lesions on withdrawal of drug. A diagnosis of ACDR was reached after exclusion of other etiologies and similar disorders like reactions due to food, infections and environmental factors. If more than one drug was thought to be responsible, the most likely offending agent was noted and the impression was confirmed by subsidence of the rash on withdrawing the drug. The causality assessment was done using WHO guidelines. The observed adverse drug reactions were classified into two principal types as per Rawlins and Thompson's classification. ${ }^{12}$ Preventability of adverse cutaneous drug reaction was assessed by Schumock and Thornton criteria. ${ }^{13}$ All the information was carefully recorded in a specially designed proforma and analysed. All the patients were educated regarding ACDRs and were given a list of drugs causing reactions for avoiding any mishap in future.

\section{RESULTS}

A total of 29073 patients attended department of dermatology during the study period. A total of 105 cases of suspected adverse cutaneous drug reactions (ACDRs) were recorded during the period of study. Out of this, 5 cases were excluded either because the offending drug was not identified or the data was insufficient to make any analysis. It was noted that the patients who were administered parenteral drugs had sudden drug eruptions than those who took oral drugs. In all, 100 cases were analyzed for ACDRs.

Out of 100 patients, $54(54 \%)$ were male and $46(46 \%)$ were female. A majority of patients $(38 \%)$ belonged to the age group of 25-44 years followed by $45-64$ years $(31 \%)$ and $15-24$ years $(10 \%)$. The types of ACDRs are elaborated in Table 1 . The commonly implicated drugs causing drug eruptions are described in Table 2.

Table 1: Types of ACDRs (based on morphology).

\begin{tabular}{|ll|}
\hline Type of drug eruption & Frequency (\%) \\
\hline Maculopapular rash & $38(38)$ \\
\hline Urticaria & $19(19)$ \\
\hline Fixed drug eruption & $12(12)$ \\
\hline SJS & $7(7)$ \\
\hline TEN & $5(5)$ \\
\hline Red-man syndrome & $5(5)$ \\
\hline Palmar-plantar erythrodysesthesia & $3(3)$ \\
\hline Angioedema & $3(3)$ \\
\hline Erythematous rash & $2(2)$ \\
\hline Erythema multiforme & $1(1)$ \\
\hline Photosensitivity & $1(1)$ \\
\hline DLE & $1(1)$ \\
\hline Others & $3(3)$ \\
\hline Total & $100(100)$ \\
\hline
\end{tabular}

ACDR: Adverse cutaneous drug reactions

SJS: Stevens Johnson syndrome, TEN: Toxic Epidermal Necrolysis, DLE: Discoid lupus erythematosus

Table 2: Commonly incriminated drugs in drug eruptions $(\mathbf{n}=\mathbf{1 0 0})$.

\begin{tabular}{|ll|}
\hline Most likely drug & Frequency $(\%)$ \\
\hline Paracetamol & $15(15)$ \\
\hline Cotrimoxazole & $10(10)$ \\
\hline Ibuprofen & $10(10)$ \\
\hline Amoxicillin & $7(7)$ \\
\hline Phenytoin & $6(6)$ \\
\hline Vancomycin & $5(5)$ \\
\hline Carbamezapine & $4(4)$ \\
\hline Total & $100(100)$ \\
\hline
\end{tabular}


Maculopapular rash $(\mathrm{n}=38)$ was found in $16(42 \%)$ males and $22(58 \%)$ females. Urticaria $(\mathrm{n}=19)$ was reported in 11 $(58 \%)$ were male and $8(42 \%)$ females. Fixed drug eruption or FDE $(n=39)$, was in $23(59 \%)$ males and 16 $(41 \%)$ females. Majority of the patients were in the age group 25- 44 years for each type of reaction. Serious adverse reactions like Stevens Johnson syndrome (SJS), Toxic epidermal necrolysis (TEN) were more common in 45-64 years and $\geq 65$ years (elderly). A case of Toxic Epidermal Necrolysis (TEN) due to Cefuroxime is shown in Figure 1. A case of Steven Johnson Syndrome (SJS) due to Carbamazepine SJS is shown in Figure 2.
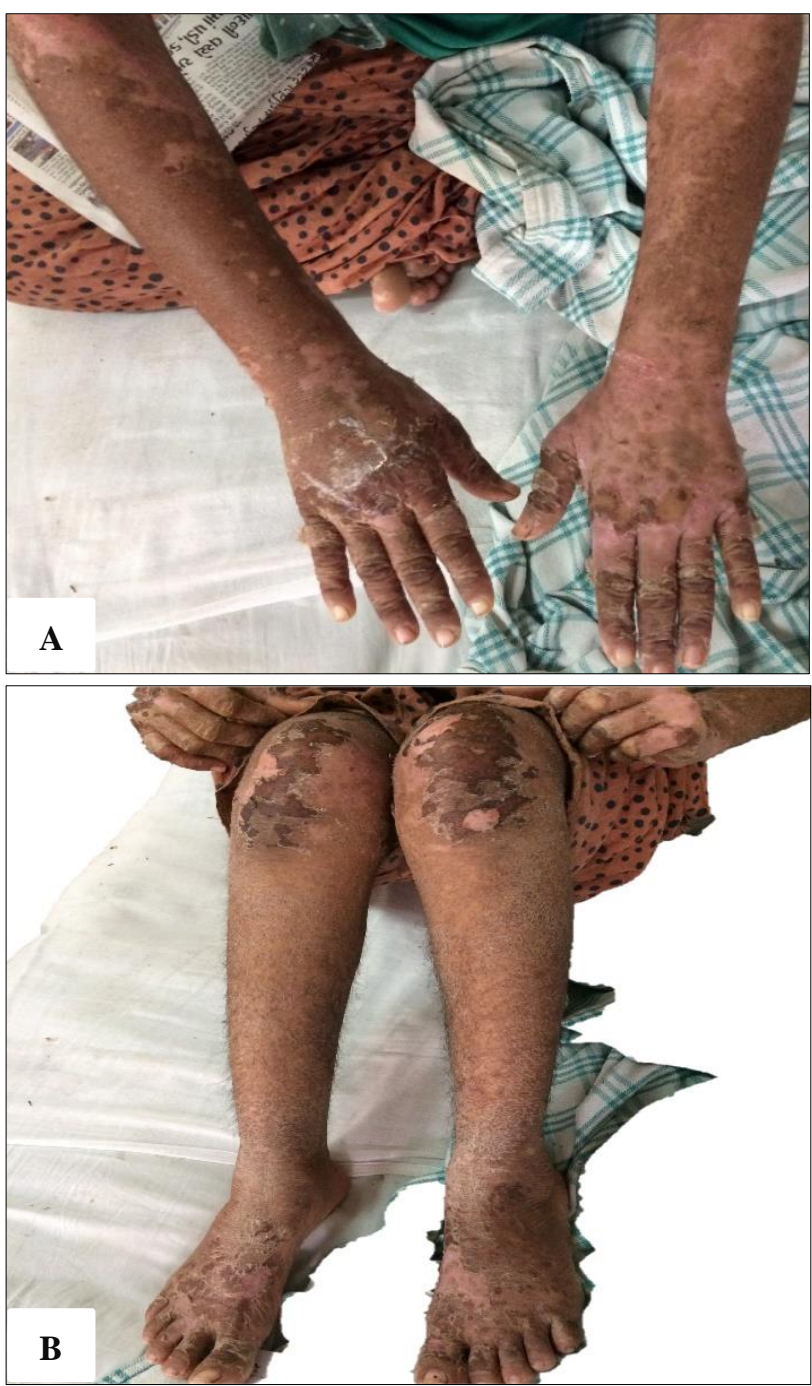

Figure 1: (A) and (B): A case of toxic epidermal necrolysis (TEN) due to cefuroxime.

The drugs responsible for FDE $(n=12)$ were NSAIDs in 9 $(75 \%)$ cases and flouroquinolones in $3(25 \%)$ cases. Mucosal involvement of ACDR $(n=100)$ was urticaria/angioedema $4(4 \%)$, FDE in $3(3 \%)$, TEN in 5 $(5 \%)$ cases and SJS in $7(7 \%)$ of the cases. The description of body surface area involvement of ACDR is shown in Table 3.

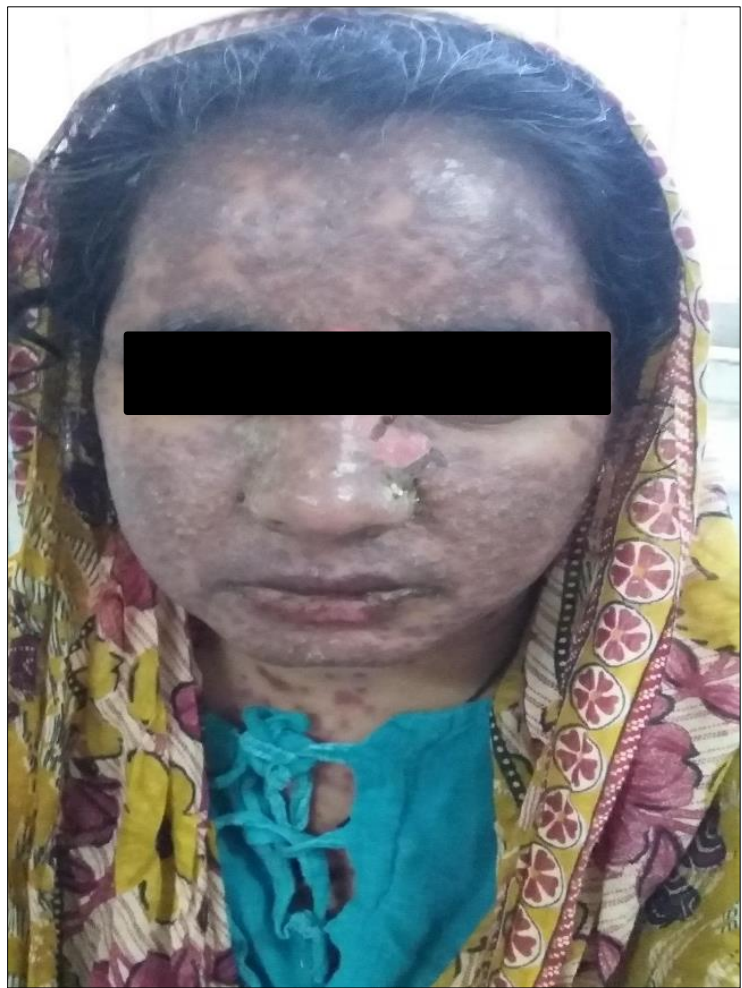

Figure 2: A case of Steven Johnson Syndrome (SJS) due to carbamazepine.

Table 3: Body surface area involvement of ACDRs.

\begin{tabular}{|ll|}
\hline Body surface area invovement & Frequency $(\%)$ \\
\hline$\leq 10 \%$ & $61(61)$ \\
\hline $11-30 \%$ & $23(23)$ \\
\hline $31-90 \%$ & $11(11)$ \\
\hline$>90 \%$ & $5(5)$ \\
\hline Total & $100(100)$ \\
\hline
\end{tabular}

ACDRs: Adverse cutaneous drug reactions

The incubation period for ADCRs $(\mathrm{n}=100)$ was less than 1 day in $23 \%$ of cases. It was between 1 to 3 days in $53 \%$ of the cases. It was less than 1 week in $11 \%$ of cases and between 1 to 3 weeks in $8 \%$ of the cases. It was more than 3 weeks in $5 \%$ of the cases. Augmented/Predictable were 7 (7\%) while bizarre/unpredictable were 93 (93\%). The causality assessment of ACDRs as per WHO-UMC criteria and Naranjo's scale is shown in Table 4.

Table 4: Causality assessment of ACDRs*.

\begin{tabular}{|lll|}
\hline $\begin{array}{l}\text { Causality } \\
\text { type }\end{array}$ & $\begin{array}{l}\text { WHO-UMC criteria } \\
\text { frequency }(\%)\end{array}$ & $\begin{array}{l}\text { Naranjo's scale } \\
\text { Frequency }(\%)\end{array}$ \\
\hline Certain & $8(8)$ & $3(3)$ \\
\hline Probable & $68(68)$ & $59(59)$ \\
\hline Possible & $24(24)$ & $383(8)$ \\
\hline Total & $100(100)$ & $100(100)$ \\
\hline
\end{tabular}

ACDRs: Adverse cutaneous drug reactions

*Cases that were unlikely, conditional or unassessable under WHO-UMC criteria were excluded from the study. 
The assessment of Severity of ACDR $(n=100)$ was mildmoderate in $94(94 \%)$ of the patients and severe $6(6 \%)$ of the patients. The Assessment of seriousness of ACDR $(n=100)$ as per WHO definition of serious adverse drug reaction was that there were $11(11 \%)$ patients who required hospitalisation (initiation or prolongation) due to ACDRs while they were life threatening in $3(3 \%)$ of the patients. Assessment of preventability of ACDR was that the ACDRs were preventable in $9(9 \%)$ of the cases, not preventable in $91(91 \%)$ of the cases.

Management of ACDR was that the suspected drug was not continued in any of the patients. Suspected drug was discontinued and symptomatic treatment was started in 67 $(67 \%)$ of cases. In 14 (14\%) of the cases, the suspected drug was discontinued and intensive medical treatment was started. The dose of suspected drug was modified in 2 (2\%) of the cases. Replacement of suspected drug was done in $17(17 \%)$ of the cases. The outcome of ACDR was that $55(55 \%)$ patients recovered completely while $5(5 \%)$ recovered with sequelae. There were $32(32 \%)$ of patients who were recovering or still under treatment. Those who continued with treatment were $6(6 \%)$ and outcome of 2 $(2 \%)$ of patients was not known.

\section{DISCUSSION}

A prospective hospital based observational study was carried out for duration of 12 months. We analysed 100 cases of ACDR and compared them with previous studies.

A majority of the patients in our study were males (M: F ratio was $1.17: 1)$. The observation is similar to other studies. ${ }^{7,14,15}$ The mean age of our patients with ACDR was $36.81 \pm 17.26$ (95\% CI). The youngest patient was 3 months and the oldest was 70 year old. This is in consonance with other Indian studies. ${ }^{7,16,17}$ Out of $15(15 \%)$ cases of serious ACDRs, $8(8 \%)$ were above 50 years of age suggesting that elderly are more likely to develop serious ACDRs. Factors which may predispose elderly to adverse drug reactions, include polypharmacy, increased potential for drug-drug interactions, age associated changes in pharmacokinetics and pharmacodynamics, pathology and use of drugs with a narrow therapeutic margin. ${ }^{18}$

The mean duration between intake of medicine and onset of rash was 6.44 days $\pm 32.48(95 \% \mathrm{CI})$ ranging from 2 hours to 154 days. These results comply with a previous study. ${ }^{9}$ Majority of adverse drug reactions $93(93 \%)$ were of Type B, since these reactions were totally aberrant effects that are not to be expected from the known pharmacological actions of a drug, when given in the usual therapeutic doses to a patient, whose body handles the drug in the normal way. The remaining 7 (7\%) ADRs belonged to Type A, since these reactions were the result of an exaggerated, but otherwise normal, pharmacological action, of a drug given in usual therapeutic doses. Another study recorded $96 \%$ Type B reactions and only $4 \%$ Type $\mathrm{A}$ in their study which is quite similar to our result. ${ }^{19}$
The most frequently reported adverse drug reactions were for antimicrobial agents in $48(48 \%)$ cases, followed by NSAIDs in $30(30 \%)$ cases and antiepileptics in $12(12 \%)$ of the cases. In the present study, among antimicrobials sulfonamides (cotrimoxazole), penicillins (ampicillin/ amoxycillin) and flouroquinolones (ofloxacin /norfloxacin/ ciprofloxacin) were the most commonly implicated drugs together accounting for almost $60 \%$ of all cases due to antimicrobials. Among NSAIDs $83 \%$ of reactions were due to ibuprofen and diclofenac sodium. Phenytoin was responsible for $50 \%$ cases due to antiepileptics followed by carbamazepine. The results were similar in other studies. ${ }^{14-17,20}$

Of the various types of adverse cutaneous drug reactions seen, maculopapular rash $(38 \%)$ was the most common $(38 \%)$ followed by urticaria $(19 \%)$ and fixed drug eruptions $(12 \%)$. These observations are in conformity with other studies. ${ }^{7,9,16,21}$ Others have noted exanthematous eruption (maculopapular rash) to be the most common type of drug reaction found fixed drug eruptions as the most common drug eruption followed by maculopapular rash and urticaria. ${ }^{20,22}$ This variation could be due to different patterns of drug usage and different ethnic group characteristics in different parts of our country.

There were 19 (19\%) patients who had mucosal involvement. They included $4(4 \%)$ cases of urticaria, 3 (3\%) cases of FDE, $5(5 \%)$ cases of TEN and $7(7 \%)$ case each of SJS and erythema multiforme. A study has reported $40 \%$ cases with mucosal involvement. ${ }^{16}$ The difference in the latter study could be explained due to increased proportion of SJS, TEN and FDE in which mucosa is more commonly involved. There were $58 \%$ patients with severe reaction had mucosal involvement as compared to $18 \%$ patients in the non severe group. A study has reported $70 \%$ and $27 \%$ mucosal involvement in the severe and non- severe group respectively. ${ }^{16}$

Antimicrobials were the most common cause of maculopapular rash in our study responsible for $48 \%$ cases followed by antiepileptics (21\%) and NSAIDS (16\%). The results are identical to a study where antiepileptics were responsible for about $8 \%$ cases. ${ }^{17}$ Our results differ from other studies where antiepileptics were the major drug groups responsible for more than $50 \%$ cases of maculopapular eruptions. ${ }^{7,9,21}$

NSAIDs and antimicrobials were the main culprits in causing urticaria. They alone were responsible for $84 \%$ cases of urticaria. Other drugs that were found to give urticarial reaction were omeprazole $(1 \%)$, bromhexine $(1 \%)$, and contrast media $(1 \%)$. These results are in accordance with earlier studies., ${ }^{4,17}$ Out of 12 cases of FDE, 7 (41\%) were due to paracetamol. Flouroquinolones were involved in $3(31 \%)$ cases followed by $2(10 \%)$ cases due to ibuprofen and aspirin. These results are in consonance with earlier studies conducted in other parts of India. ${ }^{7,8,17}$ 
Steven Johnson syndrome were recorded in 7 (7\%) of total reactions. Paracetamol was responsible in $3(3 \%)$ cases; carbamazepine $2(2 \%)$ cases and norfloxacin in $2(2 \%)$ cases. The results are similar to earlier studies conducted in India. ${ }^{15,16}$ Five $(5 \%)$ cases of Toxic epidermal necrolysis were due to carbamazepine, cotrimoxazole, ofloxacin, naproxen and cefuroxime respectively. The results comply with earlier studies. ${ }^{16,17}$

There were $5(5 \%)$ cases of red- man syndrome due to vancomycin. There were $3(3 \%)$ cases of Palmar-plantar erythrodysesthesia, 2 (2\%) cases due to cepecitabine and 1 $(1 \%)$ case due to paclitaxel. During our study, there were $3(3 \%)$ cases of angioedema due to ibuprofen (1), ampicillin (1), enalapril (1). There were $2(2 \%)$ cases of erythematous rash due to NSAIDS, $1(1 \%)$ case of erythema multiforme due to metronidazole and $1(1 \%)$ case of DLE due to phenytoin. In another study, as opposed to $6.7 \%$ cases of erythema multiforme were found. ${ }^{16}$

Ninety four (94\%) cases had mild- moderate adverse cutaneous drug reaction and they did not require any specific therapy. They were simply managed by withdrawal of the suspected drug and supportive treatment. Six $(6 \%)$ patients suffered severe adverse drug reaction and required immediate cessation of the suspected drug, hospitalization and intensive medical care. The results comply with earlier studies. ${ }^{16,19,23}$

Out of the 100 cases of ACDRs in our study, dechallenge was done in all the cases and it was positive in 84 of them. Although rechallenge was not done in any of our cases due to practical problems, these cases were considered definite because rechallenge data was available in the form of past history of similar reaction with the same drug. The difference between our study and other studies may be due to different scales used for causality assessment or because of individual differences in the interpretation of data., ${ }^{9,19}$

\section{Limitation}

The limitation of the study is that since the prescriptions were based on government medicine supply it could not assess impact of other drugs available in the market.

\section{CONCLUSION}

To conclude, monitoring and reporting of adverse drug reactions will reduce morbidity and mortality due to use of medicines. It will also promote safe and rational use of medicines amongst clinicians.

\section{ACKNOWLEDGEMENTS}

Authors would like to thank the head of the Department of Dermatology for permitting them to conduct the study. Authors are also grateful to the residents of Dermatology for helping with the clinical cases.
Funding: No funding sources

Conflict of interest: None declared

Ethical approval: The study was approved by the Institutional Ethics Committee

\section{REFERENCES}

1. International drug monitoring. Role of national centres. WHO Technical report series no. 498. Geneva, Switzerland; WHO 1972.

2. Bigby M, Jick SJ, Kien H, Amdt K. Drug-induced cutaneous reactions a report from the Boston Collaborative Drug Surveillance Program on 438 consecutive in patients 1975 to 1982 . JAMA; 1986:3358-3363.

3. Beard K. In: Lee A Eds. Adverse drug reactions, $1^{\text {st }}$ Ed, Pharmaceutical Press, London; 2001:1.

4. Nayak S, Acharjya B. Adverse cutaneous drug reaction. Indian J Dermatol. 2008:53(1):2-8.

5. Roujeau X, Huynh TN, Bracq C, Guilluame JC, Revuz J, Touraine R. Genetic susceptibility to toxic epidermal necrolysis. Arch Dermatol. 1987;123:11713 .

6. Stern RS, Wintroub BU. Cutaneous reactions to drugs. In: Freedberg IM, Eisen AZ, Wolff K, Austen KF, Goldsmith LA, Katz SI, et al editors. Fitzpatrick's Dermatology in General Medicine. $5^{\text {th }}$ Ed. New York: McGraw-Hill; 1999:1634-1642.

7. Sharma VK, Sethuraman G, Kumar B. Cutaneous adverse drug reactions: clinical pattern and causative agents-a six-year series from Chandigarh. India. J Postgrad Med. 2001;47:95-9.

8. Sehgal S, Balachandran C, Shenoi SD. Clinical study of cutaneous drug reaction in 80 patients. Indian $\mathbf{J}$ Dermatol Venereol Leprol. 2003;69:6-7.

9. Noel MV, Sushma M, Guido S. Cutaneous adverse drug reactions in hospitalized patients in a tertiary care centre. Indian J Pharmacol. 2004;36:292-5.

10. Shear NH, Knowles SR, Sullivan JR, Shapiro L. Cutaneous reactions to drugs. In: Freedberg IM, Eisen AZ, Wolff K, editors. Fitzpatrick's dermatology in general medicine. $6^{\text {th }}$ Ed. USA: McGraw Hill, Medical publishing division; 2003:1330-1336.

11. Adithan C. National pharmacovigilance programme. Indian J Pharmacol. 2005;37:347.

12. Rawlins M, Thompson W. Mechanisms of adverse drug reactions. In: Davies D, ed. Textbook of adverse drug reactions. New York: Oxford University Press. 1991:18-45.

13. Schumock G, Thornton J. Focusing on the preventability of adverse drug reactions. Hosp Pharm. 1992;27:538.

14. Mani MZ, Mathew M. A study of 218 drug eruptions. Indian J Dermatol, Venereol and Leprol. 1983;49:10917.

15. Mehta TK, Marquis L, Shelty JN. A study of 70 cases of drug eruptions. Indian J Dermatol, Venereol and Leprol. 1971;37:1-5.

16. Pudukadan D, Thappa DM. Adverse cutaneous drug reactions: Clinical pattern and causative agents in a 
tertiary care center in South India. Indian J Dermatol Venereal Leprol. 2004;70:20-4.

17. Patel RM, Marfatia YS. Clinical study of cutaneous drug eruptions in 200 patients. Indian J Dermatol Venereol Leprol. 2008;74(4):430.

18. Nolan L, O'Malley K. Adverse drug reaction in the elderly. Br J Hosp Med. 1989;41:446-57.

19. Ghosh S, Acharya LD, Rao PGM. Study and evaluation of the various cutaneous adverse drug reactions in Kasturba hospital, Manipal. Indian $\mathbf{J}$ Pharm Sci. 2006;68(2):212-5.

20. Kauppinen K, Stubb S. Drug eruptions: Causative agents and clinical types. Acta Derm Venereol. 1984;64:320-4.

21. Chatterjee S, Ghosh AP, Barbhuiya J, Dey SK. Adverse cutaneous drug reactions: A one year survey at a dermatology outpatient clinic of a tertiary care hospital. Indian J Pharmacol. 2006;38(6):429-31.

22. Sullivan JR, Shear NH. Drug eruptions and other adverse drug effects in aged skin. Clin Geriatr Med. 2002;18:21-42.

23. Sushma M, Noel MV, Ritika MC, James J, Guido S. Cutaneous adverse drug reactions: a 9-year study from a South Indian Hospital. Pharmacoepidemiol Drug Saf. 2005;14(8):567-70.

Cite this article as: Dhanani JG, Sukhlecha A. A study of adverse cutaneous drug reactions in the department of dermatology of a teaching hospital in Jamnagar, India. Int J Basic Clin Pharmacol 2017;6:2259-64. 